\title{
Influence of microhybrid resin and etching times on bleached enamel for the bonding of ceramic brackets
}

Leily Macedo Firoozmand Juliana Viana Pereira Brandão Melissa Proença Nogueira Fialho

Department of Dentistry, School of Dentistry, Univ CEUMA, São Luis, MA, Brazil.
Declaration of Interests: The authors certify that they have no commercial or associative interest that represents a conflict of interest in connection with the manuscript.

Corresponding author: Leily Macedo Firoozmand

E-mail: leilyfiroozmand@hotmail.com

Submitted: Sep 14, 2012

Accepted for publication: Dec 14, 2012

Last revision: Jan 24, 2013

\begin{abstract}
The aim of this study was to evaluate the shear bond strength (SBS) of polycrystalline ceramic brackets (PCB) bonded after bleaching treatment using different composite resins and enamel etching times. A total of 144 bovine incisors were randomly divided into two study groups ( $\mathrm{n}=72$, each) as follows: G1, enamel bleached with 35\% hydrogen peroxide, and G2 (control group), enamel unbleached. After the bleaching treatment, the samples were stored in artificial saliva for 14 days. These groups were further divided into two subgroups ( $\mathrm{n}=36$, each) as follows: GA, brackets bonded with Transbond XT (3M) and GB, brackets bonded with Filtek Z250 (3M). For each resin used, three different etching times with $37 \%$ phosphoric acid (15, 30 and 60 seconds) were tested. SBS tests were performed using a universal testing machine (EMIC), and the adhesive remnant index (ARI) score was verified. Significant differences among the three experimental conditions and interactions between the groups were observed. The type of composite resin accounted for $24 \%$ of the influence on the bond strength, whereas the etching time and bleaching treatment accounted for $14.5 \%$ and $10 \%$ of the influence on bond strength, respectively. The ARI revealed that the most common area of adhesion failure was at the composite resin-bracket interface. The type of composite resin, etching time and external bleaching significantly influenced the SBS of PCB on enamel, even after 14 days of saliva storage.
\end{abstract}

Descriptors: Tooth Bleaching; Shear Strength; Dental Enamel.

\section{Introduction}

Continuing developments in dental materials have led to improvements in contemporary dentistry. Particularly with the advent of cosmetic and conservative dentistry, studies have been undertaken to optimize adhesion using different techniques. The evolution and use of composite resins in various fields of dentistry has enabled their implementation in orthodontic practice. ${ }^{1-3}$

These resins' great aesthetic value can be observed in orthodontics with the development of aesthetic brackets, ${ }^{4,5}$ which can minimize the appearance of heavy smiles produced by metallic brackets.

Patients with bleached teeth are frequently cared for in dental offices, and bleaching can reduce the adhesion of resin materials to tooth structures. ${ }^{6}$ Various types and concentrations of bleaching agents are available for use in dental offices. ${ }^{6,7}$ Furthermore, patients have greater access to 
bleaching systems, and certain treatments are administered without professional supervision. ${ }^{7}$

The reduction in adhesive strength may be related to the ability of the resin to attack surfaces treated with hydrogen peroxide and to the effects of the bleaching agents on the resin. ${ }^{6}$ Adhesive failures are associated with areas of resin nonattachment and alterations in resin quality. ${ }^{8} \mathrm{~A}$ reliable adhesive strength is very important, and the application of forces to bleached teeth can compromise the adhesive resistance of orthodontic accessories.

Regarding the enamel surface, several authors have reported differences in adhesion values with etching times between 15 and 60 seconds, ${ }^{9,10}$ although they have agreed that 15 seconds appears to be sufficient for clinical practice. Therefore, more investigations are needed to clarify the interactions among bleaching agents, bonding techniques/materials and ceramic brackets. Although there is no consensus, ${ }^{11}$ there is evidence of significant decreases in the mean bond strength of orthodontic brackets when bonding is performed immediately after bleaching, ${ }^{6,8,12}$ and strong recommendations have been made to wait several days before undertaking any adhesive procedure. ${ }^{13-15}$

Accordingly, the purpose of this study was to determine the effects of different etching times with a bleaching treatment of $35 \%$ hydrogen peroxide on the shear bond strength of polycrystalline ceramic brackets bonded with different resins for different etching times. The null hypothesis asserted that there is no significant difference in bond strength when using different resins and enamel treatments.

\section{Methodology}

A total of 144 freshly extracted bovine incisors were used. The protocol for this experiment was approved by the Committee on Ethics and Animal Experimentation, protocol no. 40/2010. The criteria for tooth selection included teeth with enamel unaffected by any pretreatment chemical agents; with intact, unrestored and noncarious buccal surfaces and without cracks incidental to extraction.

The roots were sectioned at the cervical third and were discarded. The pulp was removed and the pulp chamber was cleaned. The pulp chamber was blotted to prevent penetration by the acrylic resin during embedding. The specimens were placed in cylindrical tubes, allowing the flat surface of the enamel to be exposed. Each tooth was oriented so that its labial surface would be parallel to the force during the shear bond test.

The specimens were randomly divided into a bleaching group (G1) and a no-bleaching group (G2, control) ( $\mathrm{n}=72$, each) and immersed in artificial saliva at $37^{\circ} \mathrm{C}$ for 14 days before bracket bonding.

The treated group was bleached with the $35 \%$ hydrogen peroxide bleaching agent Whiteness HP Maxx (FGM, Joinvile, Brazil) according to the manufacturer's instructions. The bleaching agent was applied to the enamel surface in a layer approximately $1 \mathrm{~mm}$ thick and was then exposed to an Optilight Plus LED light-curing unit (Gnatus, Ribeirão Preto, Brazil). The gel was moved periodically to release the oxygen bubbles generated and to improve the contact between the gel and the tooth. After 15 minutes, the enamel surface was rinsed with a spray of water. The bleaching treatment was repeated on each sample surface three times (45 minutes), and, after one week of storage in artificial saliva at $37^{\circ} \mathrm{C}$, the bleaching agents were reapplied, simulating the bleaching treatments made in dental clinics.

After 14 days of bleaching treatment, the treated and control groups were divided into two subgroups $(\mathrm{n}=36)$, and the enamel was cleaned with a fluoridefree pumice using a rubber polishing cup, rinsed with a water spray and dried with an oil-free air drier. In the subgroups, either Transbond XT (3M ESPE, St Paul, USA), GA, or Filtek Z250 (3M ESPE, St Paul, USA), GB, was used for the bonding of the brackets, using etching times of 15,30 or $60 \mathrm{sec}-$ onds. Twelve experimental groups were thus formed $(\mathrm{n}=12)$ (Table 1). The adhesives Primer Transbond (3M ESPE, St Paul, USA) and Adper Single Bond 2 (3M ESPE, St Paul, USA) were used for GA and GB, respectively, and were used in strict accordance with the respective manufacturers' instructions.

Polycrystalline ceramic central incisor brackets, (Orthometric Inceram, Maanshan, China) with .022" Roth - U1R, were bonded by the same operator. The bracket was bonded using a $300 \mathrm{~g}$ compressive force applied with a force gauge (Correx 
Table 1 - Experimental groups.

\begin{tabular}{|c|c|c|}
\hline $\mathrm{n}$ & $\begin{array}{l}\text { Groups according to } \\
\text { enamel bleaching and } \\
\text { composite }\end{array}$ & $\begin{array}{l}\text { Groups according to } \\
\text { etching time (seconds) } \\
(n=12 \text {, each) }\end{array}$ \\
\hline \multirow{6}{*}{72} & \multirow{3}{*}{$\begin{array}{c}\text { Bleached enamel + } \\
\text { Transbond XT }\end{array}$} & G1A - 15 \\
\hline & & G1A - 30 \\
\hline & & G1A - 60 \\
\hline & \multirow{3}{*}{$\begin{array}{c}\text { Bleached enamel }+ \\
\text { Z250 }\end{array}$} & G1B - 15 \\
\hline & & G1B - 30 \\
\hline & & G1B - 60 \\
\hline \multirow{6}{*}{72} & \multirow{3}{*}{$\begin{array}{c}\text { Non-bleached enamel + } \\
\text { Transbond XT }\end{array}$} & G2A - 15 \\
\hline & & G2A - 30 \\
\hline & & G2A - 60 \\
\hline & \multirow{3}{*}{$\begin{array}{c}\text { Non-bleached enamel + } \\
\text { Z250 }\end{array}$} & G2B -15 \\
\hline & & G2B - 30 \\
\hline & & G2B - 60 \\
\hline
\end{tabular}

Co., Haag-Streit, Bern, Switzerland) for 3 seconds, which was sufficient to seat the bracket on the tooth surface. The excess resin was removed with a scaler before photopolymerization on the mesial and distal sides of the bracket for 20 seconds each.

The specimens were stored in distilled water at $37^{\circ} \mathrm{C}$ for 24 hours, and afterwards positioned in the testing machine (DL 2000-EMIC, São José dos Pinhais, Brazil). The knife-edged shearing blade was positioned to allow the transmission of force in the occluso-gingival direction parallel to the labial surface and the bracket interface at a crosshead speed of $0.5 \mathrm{~mm} / \mathrm{min}$ without touching the enamel. The maximum load necessary to debond the bracket was recorded in megapascals and was calculated by dividing the shear bond force by the bracket area.

\section{Evaluation of residual adhesive}

After debonding, the teeth and brackets were analyzed under a stereomicroscope at $10 \times$ magnification to verify any remaining adhesive, in accordance with the adhesive remnant index (ARI). ${ }^{16}$ The ARI scores ranged from 0 to 3 as follows:

- 0 = no adherence of composite to enamel;

- $1=$ less than $50 \%$ of the composite on the enamel;

- $2=$ more than $50 \%$ of the composite on the

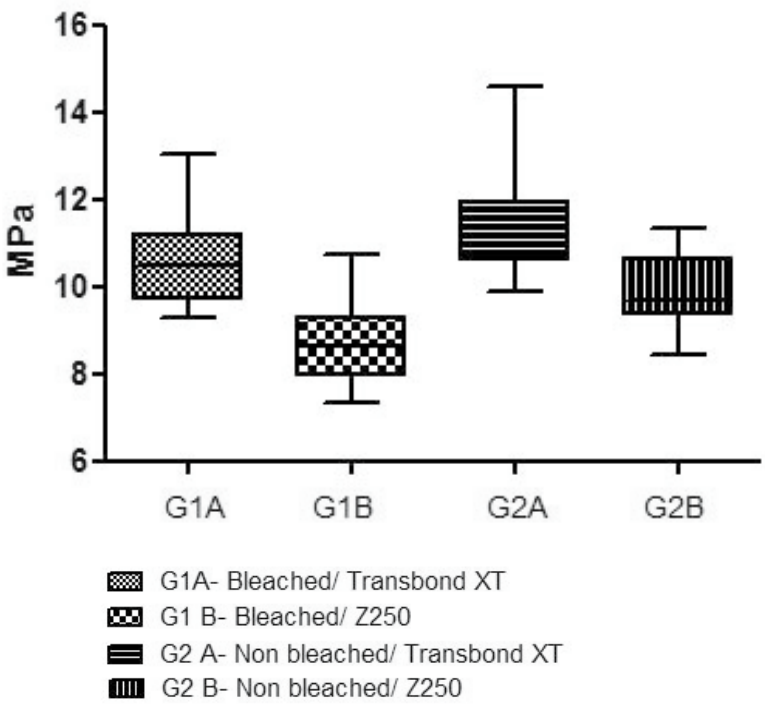

Figure 1 - Mean shear bond strengths and standard deviations for bleached and non-bleached teeth with two types of composite resin.

enamel; and

- 3 = all of the composite on the enamel, with the impression of the bracket base.

\section{Statistical analyses}

The data were subjected to three-way analyses of variance (ANOVA) and Tukey's multiple comparison statistical test $(5 \%)$ using the PASW Statistics software (v.17; SPSS Inc., Chicago, USA) and GraphPad Prism (v.5 - 2007, San Diego, USA). The chi-squared test and Spearman's correlation coefficient were used to determine the relationships between the frequency of ARI and the variables.

\section{Results}

\section{Shear bond strength testing}

The descriptive statistics for the debonding strengths under different experimental conditions of the bleached and non-bleached teeth bonded with different composites are presented in Figure 1 and Table 2.

The three-way ANOVA indicated statistically significant interactions between bleaching treatment and acid etching times $(\mathrm{F}=3.351, \mathrm{p}<0.05)$ and among the principal effects of bleaching treatment $(\mathrm{F}=14.726, \mathrm{p}<0.001)$, the type of resin $(\mathrm{F}=41.765$, 
Table 2 - Mean shear bond (standard deviations) for each study group.

\begin{tabular}{c|c|c|c|c|c}
\hline \multirow{2}{*}{} & \multicolumn{2}{|c|}{ Bleaching treatment } & \multicolumn{2}{c|}{ No bleaching treatment } & \multirow{2}{*}{ Total } \\
\cline { 2 - 5 } & Transbond XT & Z 250 & Transbond XT & \multicolumn{1}{c}{ Z 250 } & \multirow{2}{*}{} \\
\hline $15 \mathrm{~s}$ & $9.67(1.53)^{*}$ & $9.39(1.73)$ & $10.73(1.61)$ & $9.42(0.81)$ & $9.80(1.52)$ \\
\hline $30 \mathrm{~s}$ & $11.84(1.65)$ & $9.72(1.58)$ & $12.41(1.07)$ & $10.22(1.04)$ & $11.05(1.73)$ \\
\hline $60 \mathrm{~s}$ & $10.16(2.30)$ & $7.08(0.75)$ & $11.32(2.25)$ & $9.94(1.95)$ & $9.63(2.43)$ \\
\hline Total & $10.56(2.03)$ & $8.73(1.82)$ & $11.49(1.81)$ & $9.86(1.36)$ & \\
\hline${ }^{*} n=12$. & & &
\end{tabular}

Table 3 - Distribution of the adhesive remnant index scores and statistical comparisons with the results of the chi-squared test and Spearman's correlation coefficient.

\begin{tabular}{|c|c|c|c|c|c|c|c|c|c|}
\hline & \multicolumn{4}{|c|}{ ARI } & \multirow{2}{*}{$\chi^{2}$} & \multirow[b]{2}{*}{$r_{s}$} & \multirow{2}{*}{$\mathrm{p}$} & \multirow{2}{*}{ Cramer's V } \\
\hline & & 0 & 1 & 2 & 3 & & & & \\
\hline \multirow{2}{*}{ Teeth } & Bleached & 2 (2.8\%) & $5(6.9 \%)$ & 10 (13.9\%) & 55 (76.4\%) & \multirow{2}{*}{11.110} & & \multirow{2}{*}{$<0.05$} & \multirow{2}{*}{$0.251(6.3 \%)$} \\
\hline & Non-bleached & $1(1.4 \%)$ & $0(0.0 \%)$ & $4 \quad(5.6 \%)$ & 67 (93.1\%) & & & & \\
\hline \multirow{2}{*}{ Resin } & Transbond & $3(4.2 \%)$ & $4(5.6 \%)$ & 11 (15.3\%) & 54 (75.0\%) & \multirow{2}{*}{12.556} & & \multirow{2}{*}{$<0.01$} & \multirow{2}{*}{$0.276(7.6 \%)$} \\
\hline & Z250 & $0(0.0 \%)$ & $1(1.4 \%)$ & $3 \quad(4.2 \%)$ & 68 (94.4\%) & & & & \\
\hline \multirow{3}{*}{ Time } & $15 \mathrm{~s}$ & $1(2.1 \%)$ & $3(6.3 \%)$ & $3 \quad(6.3 \%)$ & 41 (85.4\%) & & \multirow{3}{*}{0.006} & \multirow{3}{*}{$>0.5$} & \multirow{3}{*}{$0.095(0.9 \%)$} \\
\hline & $30 \mathrm{~s}$ & $1(2.1 \%)$ & $1(2.1 \%)$ & $6(12.5 \%)$ & 40 (83.3\%) & & & & \\
\hline & $60 \mathrm{~s}$ & $1(2.1 \%)$ & $1(2.1 \%)$ & $5(10.4 \%)$ & 41 (85.4\%) & & & & \\
\hline
\end{tabular}

$\mathrm{p}<0.001)$, and the acid etching time $(\mathrm{F}=11.175$, $\mathrm{p}<0.001)$. The shear strength was greater in nonbleached teeth (10.67 MPa) compared with bleached teeth $(9.65 \mathrm{MPa})$, with a partial $\eta^{2}$ of 0.10 , showing that $10 \%$ of the shear bond strength was due to the bleaching treatment because the types of composites employed (Transbond XT and Z250) contributed $24 \%$ of the differences in bond strength (partial $\left.\eta^{2}=0.24\right)$. The etching time accounted for $14.5 \%$ of the influence on the adhesive strength, with the time of 30 seconds resulting in the greatest average strength (11.05 $\mathrm{MPa})$ compared with the values obtained for 15 and 60 seconds (average $=9.80 \mathrm{MPa}$ and 9.63 MPa, respectively) (Table 2).

Tukey's test demonstrated that the Transbond XT on enamel without bleaching and conditioned for 30 seconds resulted in the highest mean bond strength $(12.41 \pm 1.07 \mathrm{MPa})$.

\section{Amount of residual adhesive}

The adhesive remnant index (ARI) ${ }^{16}$ and the frequency of failure were analyzed. Even after 14 days of storage in saliva, the non-bleached group pre- sented a higher percentage of enamel-resin adhesion (score 3, 93.1\%) compared to the bleached group (76.4\%) (Table 3).

The chi-squared test results indicated significant associations between the distribution of ARI scores and the bleaching treatment $\left(\chi^{2}=9.085, \mathrm{p}<0.05\right)$, and between the ARI score and the type of composite $\left(\chi^{2}=10.978, \mathrm{p}<0.05\right)$. Spearman's correlation test showed that the etching time did not influence the ARI score frequency $\left(r_{s}=0.074, p>0.5\right)$ (Table $3)$.

\section{Discussion}

A significant number of patients believe that orthodontic appliances are unattractive and unacceptable; therefore, these patients are willing to pay more money for more aesthetically pleasing materials. In response to modern aesthetic requirements, this experimental study was undertaken to evaluate the influence of the interactions among tooth bleaching, acid etching time and type of resin on the bond strength of ceramic brackets. The null hypothesis raised in this study was rejected. 
Ceramic polycrystalline brackets based on mesh are more common and popular than monocrystalline brackets. They present similar modes of failure and bond strength; however, monocrystalline brackets are stiffer and therefore have a higher risk of fracture during removal. ${ }^{17}$ Therefore, the shear strength test was used in this research because this test is less likely to fracture the brackets than is tensile testing. ${ }^{4}$ Nevertheless, during the shear tests, fractures were observed in four polycrystalline brackets.

The treatment of the bases of ceramic brackets with abrasive/silane can lead to higher bond strength values. ${ }^{3,18}$ In several cases, values greater than $25 \mathrm{MPa}$ have been observed, ${ }^{3,18}$ at which point the chances of enamel damage are increased. ${ }^{5}$ Without ceramic base treatment, under the different conditions outlined, the average bond values obtained in this study ranged from 7.08 to $12.42 \mathrm{MPa}$. Regardless of the etching time, the use of ceramic brackets without base treatments resulted in values less than $12.75 \mathrm{MPa}$, which, as suggested by Bishara and Fehr, ${ }^{5}$ might be safer for the enamel.

Different types of resins yield results in accordance with their unique characteristics, related to their types of organic content and inorganic fillers. Modifications introduced to the composition of composite can alter their physical properties; therefore, a small modification in composition can significantly influence clinical needs. ${ }^{19}$ Composite resins used for aesthetic restorations can also be used for bonding brackets. ${ }^{2,3,20}$ Bishara et al. ${ }^{1}$ indicated that composite materials can potentially be used if their consistency can be made more fluid, so that they adhere readily to the bracket base.

Consistent with the findings of Uysal et al. ${ }^{2}$ our results showed that the Z250 (3M) exhibited lower bond strength than the Transbond XT (3M), and the type of composite used influenced $24 \%$ of the bond strength values. This result showed that among the other factors studied (bleaching treatment and etching time), the type of composite resin had the greatest influence on the bond strength. Eslamian et al. ${ }^{3}$ employed nanohybrid resins and found bond strength values higher than those presented in this study; however, their ceramic bases were previously treated.
The results of this study showed significant differences among the etching times and confirmed that bond values lower than $10 \mathrm{MPa}$ can be found when the etching time is 15 seconds. ${ }^{2}$ The etching time provided $14.5 \%$ of the difference in adhesive strength results, and the time of 30 seconds resulted in the greatest average strength, in accord with published studies. ${ }^{16,20}$

Enamel exposed to bleaching agents presents slight morphologic alterations, whereas phosphoric acid treatment causes severe morphologic alterations. ${ }^{21}$ Different types and concentrations of bleaching agents have been studied., ${ }^{72,13,22,23} \mathrm{Hy}$ drogen peroxide was chosen in this study because it can penetrate into the enamel more efficiently than carbamide peroxide as a result of its lower molecular weight. Investigations have suggested that weak bonds may result from the permanence of peroxiderelated substances, residual peroxide at or near the enamel surface, ${ }^{8}$ temporary loss of calcium from the enamel, or changes in the morphology of adamantine, ${ }^{24}$ which could be reversed by saliva through ion exchange.

In orthodontics, the influences of several bleaching systems, with various concentrations and application times, have been reported in the literature. ${ }^{16,21,22}$ However, the delay period for bonding orthodontic brackets after bleaching is controversial because although certain studies have indicated that adhesive procedures can be performed after 24 hours, ${ }^{2,21} 7$ days, ${ }^{22,23} 14$ days and 30 days, ${ }^{2}$ other studies have contradicted these results. ${ }^{24}$ An adequate time after bleaching is fundamental to normal penetration of conventional adhesive onto the enamel surface.

Contrary to previous observations, ${ }^{21}$ this study indicates that after 14 days of storage in saliva, there was a significant difference between bleached and unbleached enamel, with $10 \%$ of the influence on the shear bond strength due to the bleaching treatment. This reduction in adhesive strength after 14 days was also found by Stokes et al., ${ }^{12}$ who used hydrogen peroxide for 2 hours on enamel. It has been indicated that the bonding of orthodontic accessories must be delayed by 2-3 weeks when there is a history of dental bleaching with $38 \%$ hydrogen per- 
oxide. ${ }^{14}$

In the present study, the bleaching agent was used for a total period of 90 minutes, whereas other authors have used bleach only once for a short time in vitro, ${ }^{3}$ which might not promote effective clinical bleaching in vivo. The reduction in the adhesive bond strength after bleaching treatment appears to be time-dependent. ${ }^{8}$

Through evaluation of the ARI, it was observed that most of the failures occurred at the bracket-resin interface, in accord with previous studies. ${ }^{3,17}$ The ARI analysis corroborates the statistical results that demonstrate that the type of composite resin represents the greatest influence on the bond strength because the Z250 resin group presented a higher percentage of ARI score 3. During the removal of orthodontic accessories, failures between the resin and bracket are preferred to enamel-resin failures because lower stress is applied to the surface of the enamel. No cohesive failures of the enamel were observed after shear bond strength testing.

Further research should be undertaken to im-

\section{References}

1. Bishara SE, Ajlouni R, Soliman MM, Oonsombat C, Laffoon JF, Warren J. Evaluation of a new nano-filled restorative material for bonding orthodontic brackets. World J Orthod. 2007 Spring;8(1):8-12.

2. Uysal T, Yagci A, Uysal B, Akdogan G. Are nano-composites and nano-ionomers suitable for orthodontic bracket bonding?. Eur J Orthod. 2010 Feb;32(1):78-82.

3. Eslamian L, Borzabadi-Farahani A, Mousavi N, Ghasemi A. A comparative study of shear bond strength between metal and ceramic brackets and artificially aged composite restorations using different surface treatments. Eur J Orthod. 2012 Oct;34(5):610-7.

4. Bordeaux JM, Moore RN, Bagby MD. Comparative evaluation of ceramic bracket base designs. Am J Orthod Dentofacial Orthop. 1994 Jun;105(6):552-60.

5. Bishara SE, Fehr DE. Ceramic brackets: something old, something new, a review. Semin Orthod. 1997 Sep;3(3):178-88.

6. Titley KC, Torneck CD, Ruse ND, Krmec D. Adhesion of a resin composite to bleached and unbleached human enamel. J Endod. 1993 Mar;19(3):112-5.

7. Li Y. Safety controversies in tooth bleaching. Dent Clin North Am. 2011 Apr;55(2):255-63.

8. Torneck CD, Titley KC, Smith DC, Adibfar A. The influence of time of hydrogen peroxide exposure on the adhesion of prove the adaptation of aesthetic materials with orthodontics, thus improving their clinical application.

\section{Conclusion}

Within the limitations of this in vitro study, the following conclusions were drawn:

- The type of resin influenced the bond strength of brackets, and the Transbond XT presented a greater bond strength compared to the Z250 composite resin.

- With regard to the etching time, 30 seconds of etching provided the highest bond strength.

- After the bleaching treatment with 35\% hydrogen peroxide, the use of Transbond XT resin with an etching time of 30 seconds provided the greatest bond strength of brackets.

\section{Acknowledgements}

This study was supported by the Foundation for Research and Scientific and Technological Development of Maranhão (FAPEMA), grant no. 04/2010.

composite resin to bleached bovine enamel. J Endod. 1990 Mar;16(3):123-8.

9. Carstensen W. Clinical results after direct bonding of brackets using shorter etching times. Am J Orthod. 1986 Jan;89(1):702.

10. Kinch AP, Taylor H, Warltier R, Oliver RG, Newcombe RG. A clinical trial comparing the failure rates of directly bonded brackets using etch times of 15 or 60 seconds. Am J Orthod Dentofacial Orthop. 1988 Dec;94(6):476-83.

11. Uysal T, Basciftci FA, Uşümez S, Sari Z, Buyukerkmen A. Can previously bleached teeth be bonded safely?. Am J Orthod Dentofacial Orthop. 2003 Jun;123(6):628-32.

12. Stokes AN, Hood JA, Dhariwal D, Patel K. Effect of peroxide bleaches on resin-enamel bonds. Quintessence Int. 1992 Nov;23(11):769-71.

13. Barbosa CM, Sasaki RT, Florio FM, Basting RT. Influence of time on bond strength after bleaching with $35 \%$ hydrogen peroxide. J Contemp Dent Pract. 2008 Feb 1;9(2):81-8.

14. Mullins JM, Kao EC, Martin CA, Gunel E, Ngan P. Tooth whitening effects on bracket bond strength in vivo. Angle Orthod. 2009 Jul;79(4):777-83.

15. Patusco VC, Montenegro G, Lenza MA, Carvalho AA. Bond strength of metallic brackets after dental bleaching. Angle Orthod. 2009 Jan;79(1):122-6. 
16. Artun J, Bergland S. Clinical trials with crystal growth conditioning as an alternative to acid-etch enamel pretreatment. Am J Orthod. 1984 Apr;85(4):333-40.

17. Liu JK, Chung CH, Chang CY, Shieh DB. Bond strength and debonding characteristics of a new ceramic bracket. Am J Orthod Dentofacial Orthop. 2005 Dec;128(6):761-5.

18. Atsü SS, Gelgör IE, Sahin V. Effects of silica coating and silane surface conditioning on the bond strength of metal and ceramic brackets to enamel. Angle Orthod. 2006 Sep;76(5):857-62.

19. Bishara SE, Ajlouni R, Laffoon J, Warren J. Effects of modifying the adhesive composition on the bond strength of orthodontic brackets. Angle Orthod. 2002 Oct;72(5):464-7.

20. Isber H, Ambrosio AR, Carvalho PE, Valle-Corotti KM, Siqueira DF. Comparative in vitro study of the shear bond strength of brackets bonded with restorative and orthodontic resins. Braz Oral Res. 2011 Jan-Feb;25(1):49-55.
21. Oztas E, Bagdelen G, Kiliçoglu H, Ulukapi H, Aydin I. The effect of enamel bleaching on the shear bond strengths of metal and ceramic brackets. Eur J Orthod. 2012 Apr;34(2):232-7.

22. Bishara SE, Oonsombat C, Soliman MM, Ajlouni R, Laffoon JF. The effect of tooth bleaching on the shear bond strength of orthodontic brackets. Am J Orthod Dentofacial Orthop. 2005 Dec;128(6):755-60.

23. Dietrich AM, English J, McGrory K, Ontiveros J, Powers JM, Bussa HI Jr, et al. A comparison of shear bond strengths on bleached and unbleached bovine enamel. Tex Dent J. 2010 Mar;127(3):285-91.

24. Ernst CP, Marroquín BB, Willershausen-Zönnchen B. Effects of hydrogen peroxide-containing bleaching agents on the morphology of human enamel. Quintessence Int. 1996 Jan;27(1):53-6. 